\title{
Three-Dimensional Magnetic Resonance Imaging of the Phakic Crystalline Lens during Accommodation
}

\author{
Amy L. Sheppard, ${ }^{1}$ C. John Evans, ${ }^{2}$ Krish D. Singh ${ }^{2}$ James S. Wolffsohn, ${ }^{1}$ \\ Mark C. M. Dunne, ${ }^{1}$ and Leon N. Davies ${ }^{1}$
}

Purpose. To quantify changes in crystalline lens curvature, thickness, equatorial diameter, surface area, and volume during accommodation using a novel two-dimensional magnetic resonance imaging (MRI) paradigm to generate a complete threedimensional crystalline lens surface model.

Methods. Nineteen volunteers, aged 19 to 30 years, were recruited. $\mathrm{T}_{2}$-weighted MRIs, optimized to show fluid-filled chambers of the eye, were acquired using an eight-channel radio frequency head coil. Twenty-four oblique-axial slices of $0.8 \mathrm{~mm}$ thickness, with no interslice gaps, were acquired to visualize the crystalline lens. Three Maltese cross-type accommodative stimuli (at $0.17,4.0$, and $8.0 \mathrm{D}$ ) were presented randomly to the subjects in the MRI to examine lenticular changes with accommodation. MRIs were analyzed to generate a three-dimensional surface model.

RESULTS. During accommodation, mean crystalline lens thickness increased $(F=33.39, P<0.001)$, whereas lens equatorial diameter $(F=24.00, P<0.001)$ and surface radii both decreased (anterior surface, $F=21.78, P<0.001$; posterior surface, $F=13.81, P<0.001)$. Over the same stimulus range, mean crystalline lens surface area decreased $(F=7.04, P<$ $0.005)$ with a corresponding increase in lens volume $(F=6.06$, $P=0.005)$. These biometric changes represent a $1.82 \%$ decrease and $2.30 \%$ increase in crystalline lens surface area and volume, respectively.

Conclusions. The results indicate that the capsular bag undergoes elastic deformation during accommodation, causing reduced surface area, and the observed volumetric changes oppose the theory that the lens is incompressible. (Invest Ophthalmol Vis Sci. 2011;52:3689-3697) DOI:10.1167/ iovs.10-6805

Gor more than a decade, high-resolution magnetic resonance imaging (MRI) techniques have been available for in vitro and in vivo analysis of the accommodative structures. As issues

From the ${ }^{1}$ Ophthalmic Research Group, Life and Health Sciences, Aston University, Birmingham, United Kingdom; and ${ }^{2}$ Cardiff University Brain Research Imaging Centre, Cardiff University, Cardiff, United Kingdom.

Supported by a PhD Scholarship from Bausch \& Lomb, USA (ALS) and a College of Optometrists Research Fellowship Award, UK (LND).

Submitted for publication October 29, 2010; revised November 26, December 20, and December 29, 2010; accepted December 30 2010.

Disclosure: A.L. Sheppard, Bausch \& Lomb, USA (F); C.J. Evans, None; K.D. Singh, None; J.S. Wolffsohn, None; M.C.M. Dunne, None; L.N. Davies, None

Presented at the annual meeting of the Association for Research in Vision and Ophthalmology, Fort Lauderdale, Florida, 2009.

Corresponding author: Leon N. Davies, Ophthalmic Research Group, Aston University, Birmingham, UK B4 7ET;

1.n.davies@aston.ac.uk. such as inadequate resolution and signal-to-noise ratio (SNR) limitations have been overcome through use of higher field strengths and specialized radio frequency (RF) coils, application of this technique in accommodation and presbyopia research has become relatively more common. ${ }^{1,2}$ MRI offers several notable advantages over conventional ocular imaging techniques such as Scheimpflug photography, ultrasound biomicroscopy, and anterior segment optical coherence tomography (AS-OCT) in that images free from optical distortion may be acquired noninvasively in any desired plane. ${ }^{1,3}$ Furthermore, the entire crystalline lens and its relationship with neighboring ocular structures can be visualized.

Hitherto, the majority of published MRI studies relating to phakic accommodation have paid particular attention to the crystalline lens and ciliary muscle, and the accommodative and age-dependent changes that occur in these structures. Strenk et al. ${ }^{1}$ provided firm evidence for the first time that the human ciliary muscle maintains its in vivo contractile ability well into old age. The diameter of the ciliary muscle ring was found to reduce in response to an $8.0 \mathrm{D}$ accommodative stimulus, even in advanced presbyopes, and furthermore, a significant agedependent reduction in this diameter was identified. A later study with a larger cohort confirmed these findings and established that the accommodative and age-dependent reductions in ciliary muscle ring diameter were not affected by IOL implantation. ${ }^{4}$ The proposed persistence of ciliary muscle contractile ability in the ageing eye $\mathrm{e}^{1,5-7}$ supports the Hess-Gullstrand theory ${ }^{8,9}$ of presbyopia development, which states that lenticular changes underlie the loss of accommodation and that the ciliary muscle loses its ability to modify lens shape as a result of increased lenticular stiffness. Further studies examining ciliary muscle mobility, however, have concluded that although the ciliary body maintains its ability to move centripetally with accommodative effort, ${ }^{10}$ the forward shift of the ciliary body is lost with age. ${ }^{11-14}$

More recently, the antero-posterior mobility of the ciliary muscle apex during accommodation has been analyzed using MRI. Strenk et al. ${ }^{15}$ observed no forward accommodative movement of the muscle apex in subjects aged 22 to 91 years, contravening the widely accepted theory that the ciliary muscle moves anteriorly and inward during accommodation to reduce tension on the zonules. In addition, a case report of accommodation in a 19-year-old albino, using AS-OCT, also failed to observe a concurrent forward shift of the ciliary body with its centripetal accommodative movement. ${ }^{16}$ Conversely, a more recent AS-OCT study, using a stimulus of the same vergence $(-8.0 \mathrm{D})$ as had Strenk and colleagues, ${ }^{15}$ indicated that the ciliary muscle does move anteriorly with accommodation, by $110 \pm 86$ and $223 \pm 108 \mu \mathrm{m}$ on the nasal and temporal sides, respectively. ${ }^{17}$ Changes of this magnitude may be difficult to detect using MRI: Strenk and co-workers stated the absolute error of their technique to be $78 \mu \mathrm{m} .{ }^{15} \mathrm{~A}$ direct comparison of AS-OCT and MRI, however, may not be appro- 
priate because they may image different anatomic landmarks leading to differences in measurements. ${ }^{18}$

In addition to ciliary muscle studies, MRI has provided a wealth of information on the optical and morphologic changes in the crystalline lens with age and accommodation. Techniques to map the refractive index distribution throughout the lens have been developed ${ }^{19-21}$ and applied to human eyes in vitro $^{22}$ and in vivo. ${ }^{3,23}$ A central plateau region of high refractive index surrounded by a zone of sharp decline is present throughout life, although the peripheral reduction is steepest in older lenses. ${ }^{22,23}$ The equatorial diameter of the central plateau zone increases with age and decreases with accommodation. $^{23}$

The accommodative morphologic changes of the crystalline lens observed from two-dimensional (2-D) MRI studies to date have provided evidence to support Helmholtz's theory of accommodation. ${ }^{24}$ Several authors have documented a significant increase in lens axial thickness and reduction in equatorial diameter during accommodation. ${ }^{1,3,23}$ Jones et al. ${ }^{3}$ reported mean changes in lens thickness and equatorial diameter of $+0.050 \pm 0.024$ and $-0.067 \pm 0.030 \mathrm{~mm} / \mathrm{D}$, respectively, although these values relate to stimulus demand level, rather than objective accommodation response. Analysis of lens crosssectional area (CSA) shows an accommodative increase, with changes confined to the anterior portion of the lens. ${ }^{25}$ The increase in lens CSA with accommodation was hypothesized to be a result of lens compression in the unaccommodated state and expansion when zonular tension reduces after ciliary muscle contraction. However, changes in CSA do not necessarily correspond to a change in lens volume. ${ }^{26,27}$

Although the published MRI studies to date have undoubtedly furthered understanding of the action and ageing of accommodative structures, 2-D approaches have inherent limitations. Image acquisition planes could potentially vary between the relaxed and accommodated states, as a result of subject head movements and the convergent and excyclotorsional eye movements that accompany accommodation. Schachar et al. ${ }^{28}$ analyzed the MR images included in the publication of Jones et $\mathrm{al}^{3}$ of an eye in the relaxed and accommodated states, finding that the globe diameter was smaller in the accommodating eye. As axial globe size does not decrease during accommodation, ${ }^{29-31}$ Schachar ${ }^{1,23}$ postulated that the accommodative reduction in lens diameter observed by Jones et al. ${ }^{3}$ was due to methodological error, which would have confounded the results of previous authors. It may be unlikely that image planes would vary significantly compared to overall slice thickness (usually $3 \mathrm{~mm}$ ), but it is apparent that there is ongoing debate over the validity of 2-D methodologies to study lens equatorial diameter. Furthermore, 2-D data cannot provide direct information on lens volume. Several authors have used in vitro techniques to calculate lens volume, but in vivo measures are much more difficult to acquire. The lens is widely believed to be incompressible due to its high water content, and thus its volume would not be expected to vary during accommodation. However, Gerometta et al. ${ }^{32}$ predicted a $2.6 \%$ accommodative increase in human lens volume from a geometric model developed using published data on lenticular dimensions. Furthermore, bovine lenses have been shown in vitro to expand during simulated accommodation using a lenticular stretching device. ${ }^{33}$ A completely 3-D high-resolution MRI methodology is required to clarify in vivo changes in lens volume and equatorial diameter during human accommodation.

Singh et al. ${ }^{34}$ provided the first description of the shape of the whole eye in three dimensions from MRI data. Previous authors had only assumed a three-dimensional (3-D) shape from a limited number of 2-D slices, but the acquisition of MRI data with high resolution in all three dimensions facilitated the generation of complete 3-D surface models, providing informa- tion on ocular volume, surface area, axial length, and width. In addition to describing variations in human eye shape, the image analysis techniques described by Singh et al. ${ }^{34}$ have been applied to the developing chick eye, scanned with a 9.4 Tesla (T) small animal scanner. ${ }^{35}$ In vivo human eye studies have generally used lower field strengths (typically 1.5 or $3.0 \mathrm{~T}$ ), although Richdale et al. ${ }^{2}$ have recently reported a protocol to generate optimized 3-D images of the human eye at 7.0 T. These very high-resolution MR scans, with a voxel size of $0.1 \times$ $0.1 \times 0.5 \mathrm{~mm}$ that allowed fine structures such as the ciliary body and iris to be clearly visualized. However, the protocol required subjects to have their eyelids taped closed to reduce motion artifacts due to blinking; thus the methodology described could not be used to examine accommodative changes in ocular structures in response to visual stimuli.

To date, one published study has reported accommodated and unaccommodated lens dimensions, including volume, obtained using a 3-D MRI approach. Hermans et al. ${ }^{27}$ generated estimates of lens volume and surface area in five subjects using a $1.5 \mathrm{~T}$ scanner to acquire $\mathrm{T}_{1}$-weighted 2-D MRI scans. Application of a Canny filter to the images to determine the lens interface in eight principle meridians and calculation of volume and surface area of each of the eight segments allowed summation of these values to describe the parameters of the whole lens in three dimensions. No significant accommodative change in lens volume was observed (mean $160.1 \pm 2.8 \mathrm{~mm}^{3}$ in the relaxed state), although surface area decreased with accommodation (mean $175.9 \pm 2.5$ and $167.5 \pm 2.9 \mathrm{~mm}^{2}$ in the relaxed and fully accommodated states, respectively). The findings imply that the human crystalline lens mass is indeed incompressible, and the capsular bag undergoes elastic deformation during accommodation. However, the results should be considered with caution due to the very small cohort size, and the lack of reported repeatability data relating to the 2-D imaging and 3-D analysis techniques.

The aim of this study is to develop a 2-D MRI protocol enabling 3-D modeling of the complete crystalline lens during accommodation. The protocol will be applied to a larger cohort than the previously published 3-D study of accommodation, and intermediate stimulus levels will be explored for the first time using MRI. Changes in lenticular dimensions will be considered in relation to objectively measured accommodative response rather than stimulus demand, providing a more valid description of changes occurring in the human lens during accommodation.

\section{Methods}

Subject recruitment and preliminary data collection took place at Aston University, and all MRI work was conducted at the Cardiff University Brain Research Imaging Centre (CUBRIC). The ethics committees of both Aston University and Cardiff University approved the study, which was performed in accordance with the tenets of the Declaration of Helsinki. Written, informed consent was obtained from all participants after explanation of the nature and possible consequences of the study. Subjects consented to the initial stages of the study at Aston University and completed an MRI initial screening form to ensure suitability for the subsequent stages of the investigation. A second screening form and MRI consent form was completed at CUBRIC, immediately before scanning.

\section{MRI Protocol Development}

Generation of complete 3-D ocular surface models from 2-D MRI data has been previously described by Singh and colleagues. ${ }^{34}$ However, to apply similar analysis techniques for smaller, lenticular models, a novel scanning protocol was required, optimized for imaging the crystalline 
lens. Furthermore, it was necessary to develop a method of presenting accommodative stimuli within the confines of the scanner.

Rather than using the body coil integrated into the 3.0 T MRI system (General Electric HDx, Waukesha, WI), a birdcage-design head coil was needed to improve the SNR, which also acted as a mount for a mirror, positioned at an angle of $45^{\circ}$, to allow supine subjects to see out of the scanner. Cushioned pads were positioned between the head coil and the subject to minimize head movements. A static, highcontrast (85\%) Maltese cross target, visible through the mirror during scanning, was used for fixation, positioned on the window of the MRI control room, providing a stimulus vergence of $-0.17 \mathrm{D}$ for emmetropic subjects. Functional emmetropia was ensured for ametropic subjects by the wearing of disposable soft contact lenses (Focus Dailies or Focus Dailies Toric, nelfilcon A, 69\% water content; Ciba Vision, Duluth, GA). A static cross-target was used to avoid possible saccadic eye movements during scanning, which can cause motion artifacts. Accommodative stimulus levels of 4.0 and $8.0 \mathrm{D}$ were selected to quantify any potential dose effect. To vary accommodative demand, subjects wore a nonmetallic trial frame under the head coil, into which an occluder was placed in the left aperture and a -4.00 or $-8.00 \mathrm{DS}$ trial lens in front of the right eye. The left eye was occluded to avoid convergent eye movements, and only the right eyes of all subjects were analyzed.

Initial MRI work involved scanning sample subjects multiple times over several weeks to ascertain the ideal protocol that allowed images of sufficient resolution to be obtained while keeping scan times down to a comfortable duration. MRI parameters including bandwidth, acquisition matrix, field of view, slice thickness, number of slices, and number of signal averages were adjusted to optimize the protocol. Sagittal, axial, and coronal localizer (scout) scans were performed initially on each subject to verify the location of the crystalline lens and inform slice placement for the main scan. After the first and second major scans, subjects were moved out of the scanner to enable the trial lens to be altered to adjust stimulus vergence. A further localizer scan was therefore necessary before imaging the second and third accommodative states in case subject head movement had occurred while being moved out of, and back into, the scanner. $\mathrm{T}_{2}$-weighted images, in which the fluid-filled regions of the eye appear hyperintense, were required for analysis purposes and were collected using the vendor's fast-spin-echo (FSE) sequence, the most commonly used pulse sequence in current MRI protocols. ${ }^{36}$

The final protocol involved 24 oblique-axial slices of $0.8 \mathrm{~mm}$ thickness, with no interslice gaps, to visualize the crystalline lens fully in all three dimensions. FSE images were acquired with a bandwidth of $\pm 15.63 \mathrm{kHz}$, echo train length 24 , sequence repetition time $8580 \mathrm{~ms}$ and echo time $500 \mathrm{~ms}$. The acquisition matrix was square; $256 \times 256$, with a $205 \mathrm{~mm}$ field of view. Voxels within the images therefore had a $0.8 \mathrm{~mm}$ isotropic resolution. Three signal averages were performed, resulting in a total scan duration of 5 minutes 18 seconds. Although increasing the number of signal averages would provide improved SNR by reducing the effects of random artifacts, an unacceptable scan duration per accommodative state would have resulted from four averages. Local shimming was performed before each scan to eliminate inhomogeneities in the magnetic field around the eyes, which cause geometric distortions. ${ }^{34,37}$ Total time spent in the scanner for each subject was approximately 45-60 minutes, comprising localizer scans, main scans, and repetition of longer scans, if necessary, due to blink or motion artifacts.

The inherent motion sensitivity of the MRI protocol necessitated a means of eliminating blinking from the scans. A single blink during the main scan resulted in significant motion artifacts (due to the low number of signal averages), which would have rendered the images unsuitable for analysis. Initial experimental planning involved the use of topical corneal anesthetic (e.g., benoxinate $0.4 \%$ ) to reduce the blink reflex. Although the blink reflex is suppressed by anesthetics, it is not completely eliminated, as required for the scans. Furthermore, the Troxler effect, which causes a stimulus to disappear during prolonged viewing ${ }^{38}$ and could result in unpredictable eye movements, would not have been eliminated by topical anesthetic. A system was therefore developed to allow the scan to be paused as required for blinking and refixation. Subjects were provided with a button box, connected to a personal computer in the MRI control room, that when pressed caused the monitor to change color from gray to white, and back to gray when released. Participants could therefore alert the MRI operator to pause the scan as frequently as required for blinking and recommence once refixated on the stimulus.

\section{Main Protocol}

Nineteen pre-presbyopic volunteers ( 7 male, 12 female) aged 19 to 30 years (mean age $25.8 \pm 4.5$ years) with no previous history of ocular abnormality or intraocular surgery were recruited using general email announcements at Aston University. The inclusion criteria for "prepresbyopic" subjects was based on Millodot's description of presbyopia, ${ }^{39}$ which states that presbyopia is "a refractive condition in which the accommodative ability of the eye is insufficient for near vision work, due to ageing ... this condition usually occurs when the amplitude of accommodation has decreased to $4 \mathrm{D}$. This condition generally occurs between the age of 42 and 48 [years]" Given that all subjects were 30 years old or younger, and that they could all achieve twice the threshold suggested by Millodot (8 D), the term "pre-presbyopic" was used for the cohort in this study. Subjects with all types of refractive error were included, provided their prescription was amenable to correction with daily disposable soft contact lenses (Focus Dailies and Focus Dailies Toric, nelfilcon A, 69\% water content; Ciba Vision). The parameter ranges for these contact lenses meant subjects with spherical refractive errors less than -10.00 DS or greater than +6.00 DS were excluded from the investigation, as were those with oblique cylinders $>0.50$ DC or orthogonal cylinders $>1.50$ DC. Additionally, potential subjects were asked to complete an initial MRI screening form that excluded participants with implanted metallic devices (e.g., pacemakers, metal plates), those with possible metal fragments in the eyes or elsewhere in the body, migraine sufferers, and females who may have been pregnant, for safety reasons.

All measurements were taken from the right eye only. Refractive error was determined from the mean of five open-view distance autorefractor readings obtained with an autorefractor (Grand Seiko Auto Ref/Keratometer WAM-5500; Grand Seiko Co., Hiroshima, Japan). ${ }^{40}$ Subjects with spherical or astigmatic refractive error $>0.50 \mathrm{D}$ were corrected with disposable soft contact lenses (Focus Dailies or Focus Dailies Toric). Functional emmetropia was necessary to ensure nearidentical accommodative demand for each subject.

Objective accommodative responses were determined using the autorefractor (WAM-5500) while subjects fixated on Maltese cross targets in free space at -4 and $-8 \mathrm{D}$ stimulus vergences, presented in random order. The targets subtended a constant angular subtense of $4.6^{\circ}$. Average target luminance and Michelson contrast values were $34.0 \mathrm{~cd} / \mathrm{m}^{2}$ and $82 \%$, and $30.5 \mathrm{~cd} / \mathrm{m}^{2}$ and $80 \%$, for the 4 and $8 \mathrm{D}$ stimuli, respectively. The left eye was occluded with a patch during measurement of the response, and subjects were instructed to "carefully focus" on the center of the Maltese cross to induce both voluntary and reflex accommodation. ${ }^{41,42}$ It was ensured at this stage that participants had sufficient subjective accommodative amplitude to maintain clarity of the $8 \mathrm{D}$ stimulus, which would be required for subsequent MRI data collection. Five readings were obtained at each stimulus level, and the mean of these values was used in conjunction with the distance autorefractor results to determine the objective accommodative response. Axial lengths were obtained from the mean of five partial coherence laser interferometry readings (IOLMaster; Carl Zeiss Meditec, Dublin, CA). ${ }^{43}$ For those subjects corrected with soft contact lenses, axial length measurement was conducted at the end of initial data collection, after lens removal.

MRI data collection took place approximately 2 weeks later at CUBRIC. Participants completed a second MRI screening form to ensure none of the initial responses had changed and completed a CUBRIC consent form. While undergoing scanning, ametropic subjects 

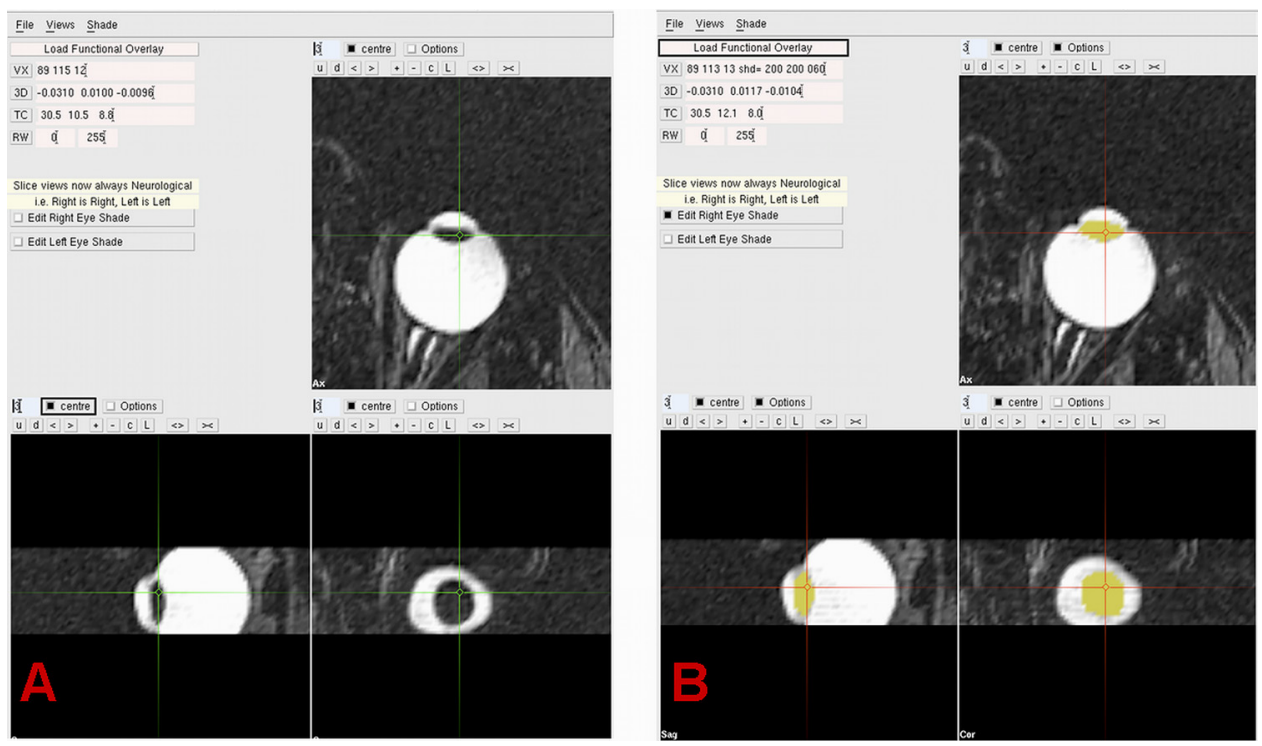

Figure 1. Image analysis (using $\mathrm{mri3dX}$ ). The lens is identified by the operator on the $\mathrm{T}_{2}$-weighted images (A), and a flood-filling algorithm is used to shade the crystalline lens voxels yellow $(\mathbf{B})$. wore contact lenses of the specification determined in the earlier stage of the study. Scans were performed according to the protocol previously described, at randomly ordered stimulus vergence levels of $-0.17,-4.0$, and $-8.0 \mathrm{D}$.

\section{MRI Data Analysis}

Image analysis was performed using a specially modified version of the freeware software package mri3dX (www.jiscmail.ac.uk/lists/ mri3dX.html) described by Singh et al. ${ }^{34}$ for semiautomated 3-D characterization of complete eye shape. The $\mathrm{T}_{2}$-weighted images, in which the fluid-filled regions of the eye appear hyperintense, are loaded into mri3dX and the dark crystalline lens manually identified (Fig. 1A). A 3-D flood-filling algorithm is used to label the voxels comprising the lens and to shade them yellow (Fig. 1B). Thresholds for voxel intensity were set at 0 to 73 for all crystalline lenses in the study after analysis of pilot MRI data from three subjects. This range was found to shade the lenticular region most accurately, although manual editing of the shaded area can be performed if necessary, in all three dimensions, to ensure complete characterization of the lens. The operator subsequently identifies the crystalline lens anterior pole with the cursor, and a sphere consisting of 32,768 triangular polygons is automatically generated to completely envelop the shaded lens voxels. An iterative shrink-wrap process is executed until the vertices of each polygon intersect a shaded voxel. The ensuing polygonal model appears ribbed, due to tight wrapping of the mesh to surface voxels; thus a rapid smoothing process is used to average local vertex positions and generate a more regular 3-D representation of the lens. The number of smoothing iterations is user defined and was set at 200 throughout the investigation as pilot analysis found this level to produce the most realistic 3-D lens models; insufficient smoothing (0-100 iterations) caused a bumpy lens surface, while excessive smoothing $(>300$ iterations) resulted in a blocky lens model.

Three-dimensional lens representations are visualized using bespoke software (iEye; Apple Computer, Cupertino, CA), allowing the model to be rotated and viewed at any orientation. The parameters of lens surface area, volume, anterior and posterior radii of curvature, axial thickness, and equatorial diameter are displayed in the system window, along with the lens model (Fig. 2). The radius of the area over which surface curvature is described is defined by the operator and was set at $3.5 \mathrm{~mm}$ for all analysis to ensure that the whole region (7.0 $\mathrm{mm}$ in diameter) fitted onto the lens model surface.

\section{Repeatability}

The repeatability of both the image analysis methodology and the complete MRI experimental protocol were investigated as this study is the first to examine the crystalline lens in three dimensions using the techniques described. First, to determine the repeatability of semiautomated image analysis with mri3dX, the images of a single, randomly selected subject at the $0.17 \mathrm{D}$ accommodative demand level were analyzed 10 times. The mean value of each of the six lens parameters was calculated, in addition to the standard deviation (SD) and standard error (SE) of the measures

To assess intersession repeatability of the entire MRI protocol, a single subject was imaged five times at the 0.17 and $4.0 \mathrm{D}$ accommodative states. Multiple repetitions of the $8.0 \mathrm{D}$ level were not feasible due to the problem of subject fatigue. While assessing repeatability, the participant was removed from the scanner after the acquisition of each long scan. Images were analyzed for the two accommodative states, and the mean, SD, and SE of each parameter were calculated.

\section{Statistical Analysis}

The relationships between axial length or refractive error and the six lens parameters measured were explored using linear regression analysis, performed with graphing and statistical software (SigmaPlot, v. 11; Systat Software, Chicago, IL). One-way repeated measures ANOVAs

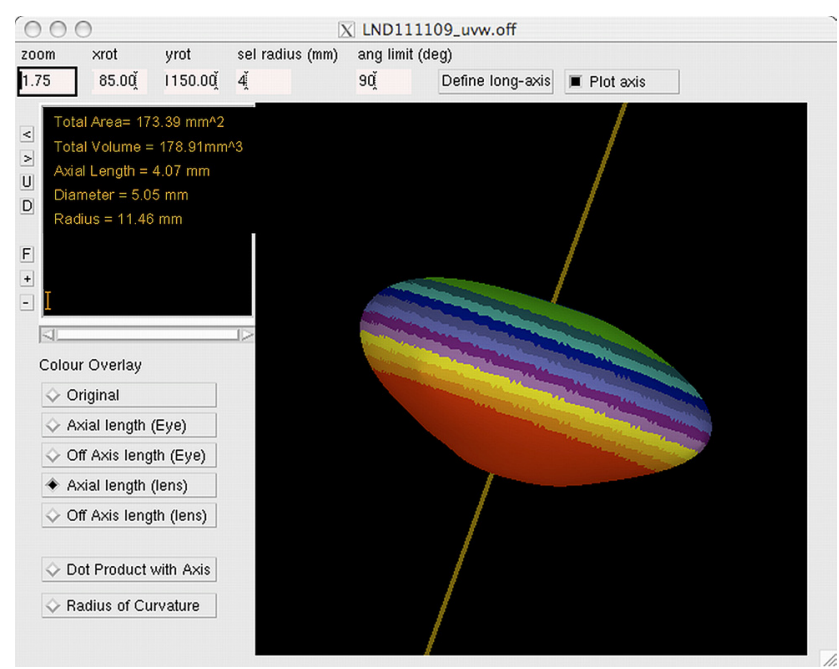

Figure 2. Visualization of 3-D lens model (using iEye software). The program allows the lens to be rotated and viewed from any orientation, in addition to providing the calculated values of area, volume, thickness, diameter, and anterior and posterior radii of curvature. 
Table 1. Repeatability of mri3dX Image Analysis Techniques, from a Single Subject Analyzed 10 Times

\begin{tabular}{ccccccc}
\hline Repeat & $\begin{array}{c}\text { Surface } \\
\text { Area } \\
\left(\mathbf{m m}^{\mathbf{2}}\right)\end{array}$ & $\begin{array}{c}\text { Volume } \\
\left(\mathbf{m m}^{\mathbf{2}}\right)\end{array}$ & $\begin{array}{c}\text { Axial } \\
\text { Thickness } \\
(\mathbf{m m})\end{array}$ & $\begin{array}{c}\text { Equatorial } \\
\text { Diameter } \\
(\mathbf{m m})\end{array}$ & $\begin{array}{c}\text { Anterior } \\
\text { Radius of } \\
\text { Curvature } \\
(\mathbf{m m})\end{array}$ & $\begin{array}{c}\text { Posterior } \\
\text { Radius of } \\
\text { Curvature } \\
(\mathbf{m m})\end{array}$ \\
\hline 1 & 172.24 & 177.00 & 4.04 & 9.94 & 13.87 & 6.32 \\
2 & 172.46 & 177.27 & 4.03 & 9.90 & 14.13 & 6.36 \\
3 & 172.46 & 177.27 & 4.04 & 10.00 & 13.82 & 6.30 \\
4 & 172.46 & 177.27 & 4.04 & 10.14 & 13.96 & 6.38 \\
5 & 172.46 & 177.27 & 4.04 & 9.92 & 13.77 & 6.42 \\
6 & 172.46 & 177.27 & 4.04 & 10.10 & 13.75 & 6.27 \\
7 & 172.46 & 177.27 & 4.02 & 9.90 & 13.86 & 6.29 \\
8 & 174.24 & 179.87 & 4.06 & 10.16 & 14.13 & 6.34 \\
9 & 172.46 & 177.27 & 4.05 & 10.14 & 13.64 & 6.31 \\
10 & 172.46 & 177.27 & 4.04 & 10.08 & 13.47 & 6.22 \\
Mean & 172.62 & 177.50 & 4.04 & 10.03 & 13.84 & 6.32 \\
SD & 0.58 & 0.84 & 0.01 & 0.11 & 0.20 & 0.06 \\
SE & 0.18 & 0.26 & 0.01 & 0.03 & 0.07 & 0.02 \\
\hline
\end{tabular}

using commercially available statistical software (SPSS v. 15; SPSS, Chicago, IL) were used to determine whether accommodation caused significant changes in lens biometric characteristics. Demand, the within-subjects factor, was assigned three levels; $0.17,4.0$, and $8.0 \mathrm{D}$. A significance level of $\alpha=0.05$ was used throughout analysis.

\section{RESUlts}

\section{Repeatability}

The results obtained from analyzing a single image set ten times are displayed in Table 1 , while the intersession repeatability data of the complete MRI technique is shown in Table 2. Both the image analysis and complete MRI techniques appear robust, with intersession SD and SE values for lens thickness, diameter, and anterior and posterior radii being within the voxel size of the original scans. Interestingly, the SDs and SEs of lens parameters are very similar at the 0 and $4 \mathrm{D}$ levels (Table 2), suggesting that subject fatigue in the accommodated state is not of significant detriment to the results. Use of the button box to pause scanning as necessary may reduce fatigue, providing more consistent data.

\section{Main Investigation}

Mean spherical equivalent (MSE) refractive error of the 19 participants ranged from -6.00 to $+0.19 \mathrm{D}$ (mean $-2.38 \pm 2.09 \mathrm{D})$. Mean objective accommodative responses to the 4.0 and $8.0 \mathrm{D}$ stimuli were $2.91 \pm 0.66$ and $5.65 \pm 1.12 \mathrm{D}$, respectively. Images of sufficient clarity for analysis purposes were obtained from all subjects at the three stimulus levels, although occasional motion artifacts required a proportion of scans (approximately 15\%) to be repeated, during the same session.

Across the whole cohort, mean unaccommodated lens thickness was $3.75 \pm 0.35 \mathrm{~mm}$, which significantly increased with accommodation $(F=33.39, P<0.001)$ at both of the accommodative vergence levels. From the 0.17 to $4.0 \mathrm{D}$ level, mean change in lens thickness was $+0.06 \pm 0.08 \mathrm{~mm}$ per diopter of accommodative response $\left(\mathrm{D}_{\text {Resp }}\right)$, while between the 4.0 and $8.0 \mathrm{D}$ levels, the increase was $0.09 \pm 0.12 \mathrm{~mm} /$

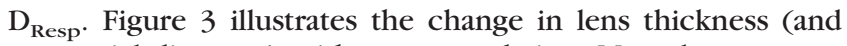
equatorial diameter) with accommodation. Mean lens equatorial diameter was $9.49 \pm 0.45 \mathrm{~mm}$ in the relaxed state, with a significant accommodative reduction observed $(F=24.00, P<$ 0.001 ), which was greatest between the 0.17 and $4.0 \mathrm{D}$ levels. Between the 0.17 and $4.0 \mathrm{D}$ levels, mean change in lens equatorial diameter was $-0.14 \pm 0.17 \mathrm{~mm} / \mathrm{D}_{\text {Resp }}$, but between the 4.0 and $8.0 \mathrm{D}$ levels the mean change was much smaller, at $-0.01 \pm 0.16 \mathrm{~mm} / \mathrm{D}_{\text {Resp. }}$. No significant correlation was identified between unaccommodated lens thickness or equatorial diameter and axial length or refractive error.

Regarding surface curvatures, the posterior lens surface was steeper at all three demand levels. In the relaxed state, mean anterior and posterior radii of curvature were $11.89 \pm 2.75$ and $6.12 \pm 0.75 \mathrm{~mm}$, respectively. Linear regression analysis identified a significant negative correlation between MSE refractive error and anterior lens radius of curvature $(R=0.53, P=$ $0.02)$, but no such relationship was present for the posterior lens radius $(R=0.09, P=0.71)$. Furthermore, neither the anterior or posterior radius of curvature demonstrated a correlation with axial length $(R=0.08, P=0.75 ; R=0.19, P=$ 0.44 , for the anterior and posterior surfaces, respectively).

TABLE 2. Intersession Repeatability of Complete MRI Technique

\begin{tabular}{lrccccc}
\hline & $\begin{array}{c}\text { Surface } \\
\text { Area } \\
\left(\mathbf{m m}^{\mathbf{2}}\right)\end{array}$ & $\begin{array}{c}\text { Volume } \\
\left(\mathbf{m m}^{2}\right)\end{array}$ & $\begin{array}{c}\text { Axial } \\
\text { Thickness } \\
(\mathbf{m m})\end{array}$ & $\begin{array}{c}\text { Equatorial } \\
\text { Diameter } \\
(\mathbf{m m})\end{array}$ & $\begin{array}{c}\text { Anterior } \\
\text { Radius of } \\
\text { Curvature } \\
(\mathbf{m m})\end{array}$ & $\begin{array}{c}\text { Posterior } \\
\text { Radius of } \\
\text { Curvature } \\
(\mathbf{m m})\end{array}$ \\
\hline Mean: 0 D & 172.45 & 177.45 & 3.95 & 9.78 & 11.18 & 6.42 \\
SD & 1.45 & 1.11 & 0.08 & 0.09 & 0.61 & 0.40 \\
SE & 0.73 & 0.56 & 0.04 & 0.04 & 0.31 & 0.20 \\
Mean: 4 D & 168.17 & 177.35 & 4.09 & 9.60 & 10.20 & 5.95 \\
SD & 2.11 & 1.45 & 0.09 & 0.12 & 0.58 & 0.32 \\
SE & 0.95 & 0.65 & 0.04 & 0.05 & 0.26 & 0.14 \\
\hline
\end{tabular}

A single subject was scanned five times at the $0 \mathrm{D}$ and $4 \mathrm{D}$ stimulus levels, and images were analyzed with mri3dX. 


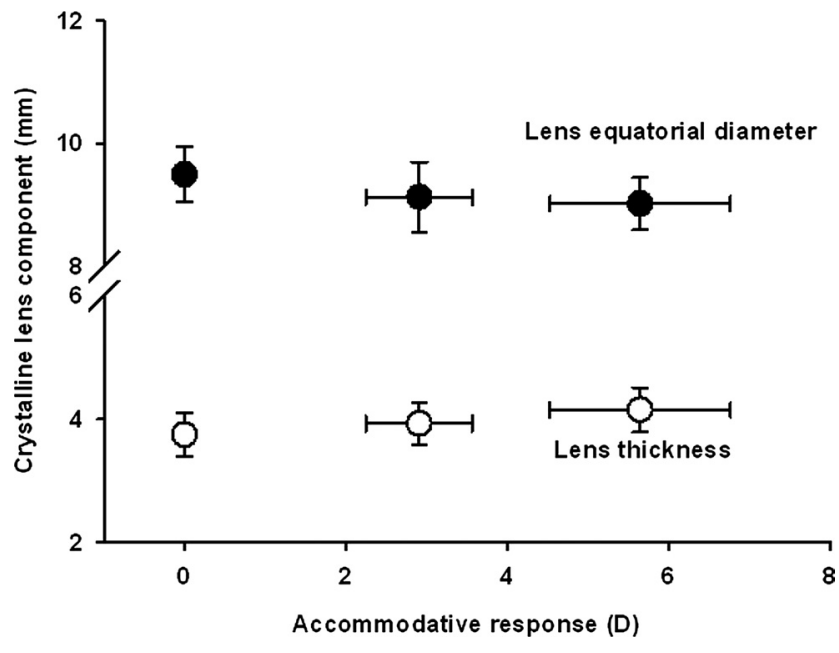

Figure 3. Change in lens equatorial diameter (closed symbols) and lens thickness (open symbols) with accommodation. $n=19$ young subjects. Error bars, $\pm 1 \mathrm{SD}$. A statistically significant reduction in lens equatorial diameter and increase in lens thickness were observed with accommodation.

Both lens surfaces, but particularly the anterior surface, showed a significant reduction in radius with accommodation $(F=21.78, P<0.001 ; F=13.81, P<0.001$, for anterior and posterior surfaces, respectively). Mean reductions in anterior radius were $0.82 \pm 1.04 \mathrm{~mm} / \mathrm{D}_{\text {Resp }}$ between the 0.17 and $4.0 \mathrm{D}$ stimulus levels, and $0.63 \pm 1.19 \mathrm{~mm} / \mathrm{D}_{\text {Resp }}$ between the 4.0 and 8.0 D levels. The posterior lens surface curvature steepened by $0.15 \pm 0.23 \mathrm{~mm} / \mathrm{D}_{\text {Resp }}$ and $0.46 \pm 1.45 \mathrm{~mm} / \mathrm{D}_{\text {Resp }}$ between the 0.17 to 4.0 and 4.0 to $8.0 \mathrm{D}$ levels, respectively. Figure 4 illustrates the change in anterior and posterior lens surface curvature with accommodation.

Mean lens surface area in the relaxed state was $157.72 \pm 11.59 \mathrm{~mm}^{2}$, which reduced significantly with accommodation $(F=7.04, P=0.003)$. Between the 0.17 and $4.0 \mathrm{D}$ levels, lens surface area reduced on average by $4.75 \mathrm{~mm}^{2}$, which represents a decrease of $1.73 \pm 2.01 \mathrm{~mm}^{2} / \mathrm{D}_{\text {Resp }}$. Between 4.0 and $8.0 \mathrm{D}$, a small increase in lens surface area was measured, $0.69 \pm 3.27 \mathrm{~mm}^{2}$, although a paired $t$-test indicated

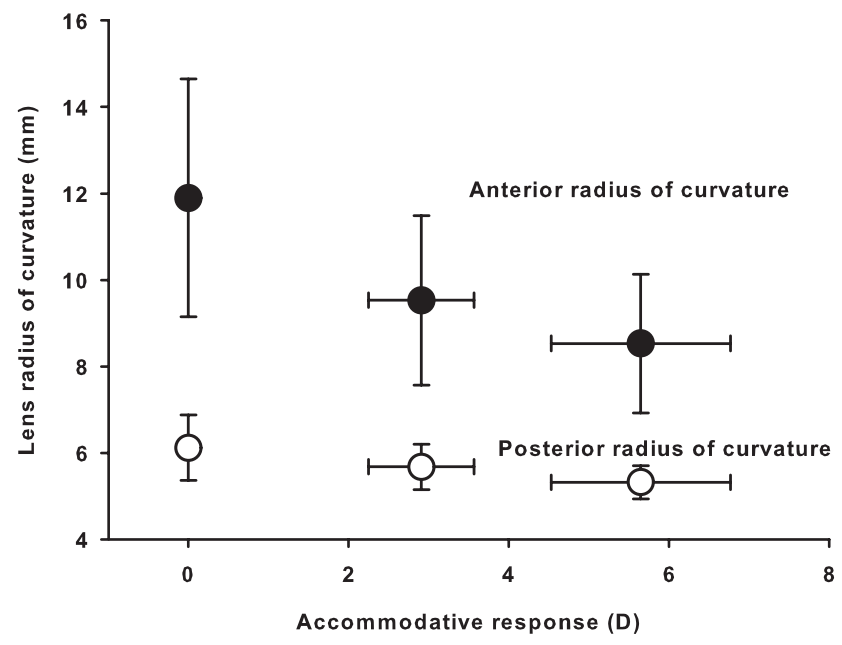

Figure 4. Change in anterior (closed symbols) and posterior (open symbols) lens surface radii of curvature during accommodation. Error bars, \pm 1 SD. A statistically significant reduction in both surface curvatures was identified during accommodation, although the anterior lens surface showed the greatest steepening.



FIGURE 5. Change in lens surface area with accommodation. Error bars, \pm 1 SD. Overall, a significant reduction in surface area was observed with accommodation, although the change consisted of a large, significant reduction between 0.17 and $4.0 \mathrm{D}$, accompanied by a small and insignificant increase between the 4.0 and $8.0 \mathrm{D}$ levels.

that this change was not statistically significant $(t=-0.41, P=$ 0.68 ). Figure 5 shows the change in lens surface area with accommodation.

Although lens surface area demonstrated an overall reduction with accommodation, mean lens volume increased significantly $(F=6.06, P=0.005)$, from $154.52 \pm 19.00 \mathrm{~mm}^{3}$ in the relaxed state to $158.08 \pm 22.89 \mathrm{~mm}^{3}$ at $8.0 \mathrm{D}$ stimulus vergence, a mean change of $+0.65 \pm 1.62 \mathrm{~mm}^{3} / \mathrm{D}_{\text {Resp }}$. The overall mean increase in volume from the relaxed state to $8.0 \mathrm{D}$ level was composed of a large increase between the 4.0 and 8.0 D stimulus levels, $2.36 \pm 5.71 \mathrm{~mm}^{3} / \mathrm{D}_{\text {Resp }}$, which was preceded by a nonsignificant $(t=1.73, P=0.11)$ reduction in volume of $-1.24 \pm 3.13 \mathrm{~mm}^{3} / \mathrm{D}_{\text {Resp }}$ from the relaxed state to the $4.0 \mathrm{D}$ level. Figure 6 illustrates the change in lens volume measured during accommodation.

\section{Discussion}

The crystalline lens accommodative changes observed are in accordance with the widely accepted Helmholtzian theory. A

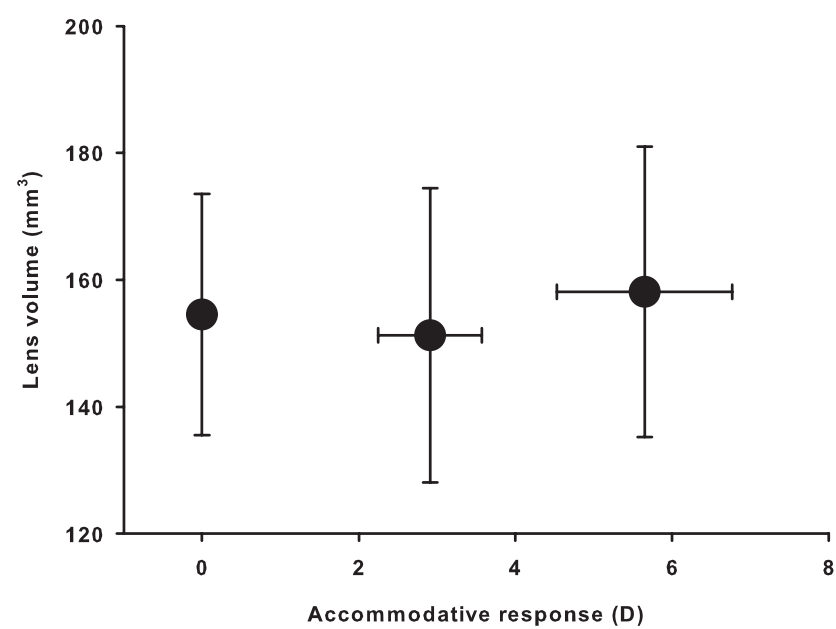

FIGURE 6. Change in lens volume with accommodation. Error bars, $\pm 1 \mathrm{SD}$. A significant increase in lens volume with accommodation was identified, but this change was composed of a nonsignificant reduction in volume from the 0.17 to $4.0 \mathrm{D}$ levels and a significant increase from the 4.0 to $8.0 \mathrm{D}$ levels. 
statistically significant accommodative increase in lens thickness and reduction in equatorial diameter was accompanied by a steepening of surface curvatures. The increase in lens thickness with accommodation has been well documented previously, using in vivo imaging techniques including MRI, 1,3,23,27 Scheimpflug photography, ${ }^{44}$ AS-OCT, ${ }^{45}$ and ultrasonography. ${ }^{46}$ However, the inability to view the equatorial region of the lens, due to the presence of the iris, has hindered in vivo determination of the effect of accommodation on human lens diameter. Previous 2-D MRI analysis studies reporting a reduction in lens equatorial diameter during accommodation ${ }^{1,3}$ have received criticism for the possibility that the lenticular changes observed were due to differing acquisition planes rather than accommodative state. ${ }^{28,47}$ According to the theory postulated by Schachar, ${ }^{48,49}$ accommodation causes the lens equator to move outward toward the sclera, secondary to increased equatorial zonular tension. However, the results of the present 3-D study provide evidence in direct opposition to Schachar's theory. Between the 0.17 and $8.0 \mathrm{D}$ stimulus levels, the mean change in equatorial diameter with accommodation was $-0.09 \pm 0.07 \mathrm{~mm} / \mathrm{D}_{\text {Resp, }}$ representing an overall mean reduction of $4.95 \%$.

The MRI protocol enabled anterior and posterior lens surface curvatures to be measured without the need to correct for optical distortion. A significant negative correlation $(R=0.53$, $P=0.02$ ) was identified between anterior lens radius of curvature and refractive error (i.e., higher myopes have flatter anterior lens surfaces). No such relationship was found regarding the posterior lens surface. Previous authors have identified no dependence of anterior lens radius on refractive error using phakometry ${ }^{50}$ and corrected Scheimpflug imaging. ${ }^{51}$ However, the crystalline lens is known to become more steeply curved with age, ${ }^{51-53}$ so it is possible that any relationship between anterior lens curvature and refractive error would be most apparent in young subjects, such as the participants in this study. Further investigation using alternative imaging methodology, to allow a greater volume of data to be collected, is required to confirm a link between anterior lens radius and refractive error and explore the cause of any relationship.

The observed decreases in lens radii of curvature with accommodation were most significant for the anterior lens surface, although the posterior surface remained steeper at all stimulus levels. Previous authors have described similar accommodative changes in lens surface curvatures theoretically ${ }^{54}$ and empirically using Scheimpflug imaging ${ }^{44,55}$ and phakometry. ${ }^{46,56}$ The present study indicates that 3-D MRI modeling represents a further methodology for analysis of changes in lens radii during accommodation. The measured curvature changes were nonlinear in nature, particularly for the posterior lens surface. Between the 0.17 and $4.0 \mathrm{D}$ levels, the anterior and posterior radii decreased by $0.82 \pm 1.04$ and $0.15 \pm 0.23$ $\mathrm{mm} / \mathrm{D}_{\text {Resp }}$, respectively, while from the 4.0 to the $8.0 \mathrm{D}$ levels, there was a small reduction in the anterior surface response, to $-0.63 \pm 1.19 \mathrm{~mm} / \mathrm{D}_{\text {Resp, }}$, but a threefold increase in the posterior radius change, to $-0.46 \pm 1.45 \mathrm{~mm} / \mathrm{D}_{\text {Resp. }}$ Previous ASOCT data regarding lenticular surface position during accommodation (Davies LN, et al. IOVS 2008;49:ARVO E-Abstract 3777) has suggested that significant accommodative changes in the posterior lens surface occur only above the $1.5 \mathrm{D}$ response level; before this, lenticular changes are limited to the anterior portion of the lens. Further investigation is required to confirm the nature of the accommodative response of the posterior lens surface, through application of techniques such as ASOCT, phakometry, and Scheimpflug imaging to a substantial cohort of young subjects, with high amplitudes of accommodation, at a range of intermediate vergence levels.

The creation of fully 3-D lens models allowed determination of in vivo lens surface area and volume changes during accom- modation in the largest cohort described to date. A significant overall reduction in surface area $(P=0.003)$ and an increase in lens volume ( $P=0.005)$ was observed. A mean $4.78 \%$ accommodative decrease in lens surface area but maintenance of constant lens volume was measured by Hermans et al. ${ }^{27}$ with MRI data from five subjects. The present study has identified a mean reduction in lens surface area of $1.82 \%$ from the relaxed state to the $8.0 \mathrm{D}$ stimulus, with an increase in volume of $2.30 \%$. The results suggest that the capsular bag undergoes elastic deformation during accommodation, causing reduced surface area, while the volumetric changes oppose the theory that the lens is incompressible due to its high water content. During distance viewing, when the zonules are taut, the lens appears to be relatively compressed, with expansion occurring when zonular tension reduces during accommodation. The data support the assertion that lens mechanics cannot be modeled as a simple incompressible fluid-filled $\operatorname{sac}^{57}$ and validate previous MRI studies examining crystalline lens CSA changes with accommodation. ${ }^{25}$ The observed $2.3 \%$ increase in lens volume is in accordance with the theoretical $+2.6 \%$ accommodative change calculated by Gerometta et al., ${ }^{32}$ based on geometric modeling of the human lens. The dual occurrence of increased volume and reduced surface area has been predicted previously by Zamudio et al. ${ }^{33}$ by further mathematical modeling of mammalian and human lenses, based on their elliptical forms. The present study is the first to provide in vivo support of the theory developed by Gerometta et al. ${ }^{32}$ and modified by Zamudio et al. ${ }^{33}$

For comparison purposes, the results of the present study are summarized in Table 3, along with recent in vivo studies that have applied MRI and alternative imaging techniques to measure changes in lens parameters with accommodation. It is apparent that the unaccommodated lens dimensions reported here compare well with previously published data, providing further evidence for the validity of the novel MRI acquisition and image analysis techniques described. Furthermore, the measured changes in lens thickness, equatorial diameter, and radii of curvature are very similar to those documented by earlier studies that have reported differences based on accommodative response, rather than stimulus demand. Only one previous study has described in vivo lens surface area and volume characteristics. Hermans et al. ${ }^{27}$ reported larger values for both mean surface area $(175.9 \pm 2.8$ versus $157.7 \pm 11.6$ $\left.\mathrm{mm}^{2}\right)$ and lens volume $\left(160.1 \pm 2.5\right.$ versus $\left.154.5 \pm 19.0 \mathrm{~mm}^{3}\right)$ than the present investigation, with lower associated standard deviations, a possible consequence of the smaller sample size. No alternative technique to MRI, for validation of the measures, is currently available for measurement of lens volume and surface area in vivo.

While evaluating the results and implications of the present study, it is also important to consider the limitations of the protocol. The strong field strength used in MRI, preventing use of metallic machinery in the scanning room, and the physical limitations of the scanner borehole prohibit measurement of accommodative response during image acquisition. Accommodative responses to the 4.0 and $8.0 \mathrm{D}$ stimuli were therefore determined before the MRI session, with the subject sitting upright. The supine scanning position, however, may reduce accommodative amplitude by over a diopter due to backward movement of the accommodated lens under gravity. ${ }^{58,59} \mathrm{Al}-$ though it may be technically difficult to objectively record the accommodative response of a supine subject, future studies should ensure that participants have sufficient subjective amplitude to maintain clarity of the stimuli while lying down, with the head facing upward.

Although the results compare favorably with previously published data, the resolution of the technique is limited by the $0.8 \mathrm{~mm}$ isotropic voxel size, which was necessary to develop 
TABle 3. Mean Values ( \pm Standard Deviation) of the Six Lens Parameters Measured in the Current Investigation and the Change in These Parameters with Accommodation, Assuming a Linear Response Profile from the 0.17 to 8.0 D Levels

Mean Value in Relaxed State and Accommodative Change Reported Previously

\section{Mean Value at $0.17 \mathrm{D}$ and Accommodative Change Observed in Present}

Lens Parameter Study

$3.75 \pm 0.35 \mathrm{~mm}$ $+0.08 \pm 0.05 \mathrm{~mm} / \mathrm{D}_{\text {Resp }}$

Equatorial diameter

$9.49 \pm 0.45 \mathrm{~mm}$ $-0.09 \pm 0.065 \mathrm{~mm} / \mathrm{D}_{\text {Resp }}$

Anterior radius of curvature

$11.89 \pm 2.75 \mathrm{~mm}$ $-0.63 \pm 0.50 \mathrm{~mm} / \mathrm{D}_{\text {Resp }}$

Posterior radius of curvature

$6.12 \pm 0.75 \mathrm{~mm}$ $-0.15 \pm 0.18 \mathrm{~mm} / \mathrm{D}_{\text {Resp }}$ Surface area $157.72 \pm 11.59 \mathrm{~mm}^{2}$ $-0.54 \pm 1.08 \mathrm{~mm}^{2} / \mathrm{D}_{\text {Resp }}$ $154.52 \pm 19.0 \mathrm{~mm}^{3}$ $+0.65 \pm 1.62 \mathrm{~mm}^{3} / \mathrm{D}_{\text {Resp }}$

Volume
Accommodative Change and Study Type/Reference

$$
\begin{aligned}
3.66 \pm 0.14 \mathrm{~mm} \\
3.78 \pm 0.22 \mathrm{~mm} \\
4.05 \pm 0.20 \mathrm{~mm} \\
3.73 \mathrm{~mm}^{*} \\
3.63 \pm 0.07 \mathrm{~mm} \\
3.70 \pm 0.10 \mathrm{~mm} \\
3.63 \pm 0.26 \mathrm{~mm} \\
9.58 \pm 0.26 \mathrm{~mm} \\
9.12 \pm 0.33 \mathrm{~mm} \\
9.33 \pm 0.33 \mathrm{~mm} \\
9.21 \pm 0.29 \mathrm{~mm} \\
11.45 \pm 1.7 \mathrm{~mm} \\
12.3 \pm 0.8 \mathrm{~mm} \\
11.25 \pm 0.4 \mathrm{~mm} \\
6.11 \pm 1.4 \mathrm{~mm} \\
6.10 \pm 0.2 \mathrm{~mm} \\
175.9 \pm 2.8 \mathrm{~mm} \\
\\
160.1 \pm 2.5 \mathrm{~mm}^{3}
\end{aligned}
$$

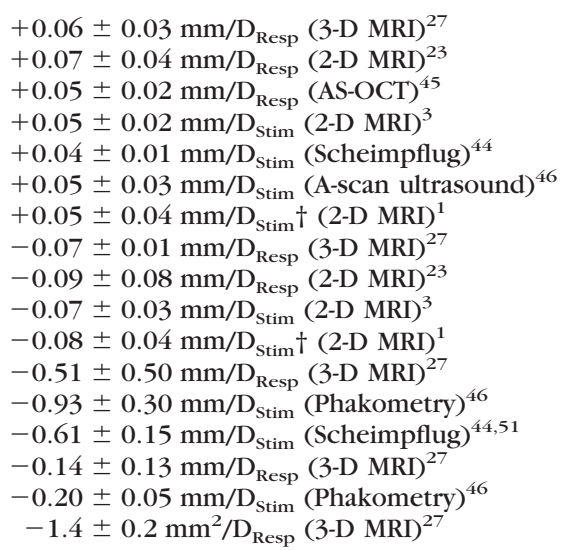

No significant accommodative change (3-D MRI) ${ }^{27}$

Previously published data are provided for comparison. $\mathrm{D}_{\text {Resp }}$ and $\mathrm{D}_{\mathrm{Stim}}$ are diopters of accommodative response and diopters of stimulus demand, respectively.

* Based on linear regression results, for a 25 -year-old subject.

† Based on reported values for the 10 youngest participants (aged 22-31 years).

the novel 3-D analysis paradigm. Image acquisition with 0.7 $\mathrm{mm}$ voxels reduced the SNR unacceptably for data analysis purposes. Image quality, and potentially variability of the results, could have been improved by increasing the number of signal averages to four, but the resultant scan length per accommodative state was too taxing for participants and resulted in greater variability. Thus, a compromise in image quality was needed to limit acquisition times. Richdale et al. ${ }^{2}$ have recently described 7.0 T 3-D imaging of the human eye in vivo with superior resolution and visualization of the crystalline lens, although the scanning protocol requires subjects to have their eyelids taped closed to minimize motion artifacts, thus preventing the viewing of accommodative stimuli.

In conclusion, this study is the largest to provide data on crystalline lens biometric characteristics, in the relaxed and accommodated states, using an in vivo 3-D MRI methodology. The accommodative changes observed support the classic Helmholtzian theory of accommodation and are in agreement with the findings of previous 2-D MRI investigations. An overall decrease in lens surface area and increase in lens volume with accommodation have been observed for the first time in vivo. The findings suggest that the lens material is compressed in the disaccommodated state and undergoes expansion when zonular tension reduces secondary to ciliary muscle contraction.

\section{References}

1. Strenk SA, Semmlow JL, Strenk LM, Munoz P, Gronlund-Jacob J, De Marco J. K. Age-related changes in human ciliary muscle and lens: a magnetic resonance imaging study. Invest Ophthalmol Vis Sci. 1999; $40: 1162-1169$.

2. Richdale K, Wassenaar P, Teal Bluestein, K, et al. 7 Tesla MR imaging of the human eye in vivo. J Magn Reson Imaging. 2009; 30:924-932.
3. Jones CE, Atchison DA, Pope JM. Changes in lens dimensions and refractive index with age and accommodation. Optom Vis Sci. 2007;84:990-995.

4. Strenk SA, Strenk LM, Guo S. Magnetic resonance imaging of aging, accommodating, phakic, and pseudophakic ciliary muscle diameters. J Cataract Refract Surg. 2006;32:1792-1798.

5. Sheppard AL, Davies LN. The effect of ageing on in vivo human ciliary muscle morphology and contractility. Invest Ophthalmol Vis Sci. 2011;52:1809-1816.

6. Pardue MT, Sivak JG. Age-related changes in human ciliary muscle. Optom Vis Sci. 2000;77:204-210.

7. Park KH, Yun JH, Kee C. The effect of cataract extraction on the contractility of ciliary muscle. Am J Ophthalmol. 2008;146:8-14.

8. Hess C. Arbeiten aus Gebiete der Akkommodationslehr. Graefes Arch Clin Exp Ophthalmol. 1896;41:1.

9. von Gullstrand A. Mechanism of accommodation. In Handbuch der Physiologischen Optik, vol. 1 City: Optical Society of America; 1924;1:382- 415 .

10. Croft MA, McDonald JP, James RJ, et al. Surgical intervention and accommodative responses I: centripetal ciliary body, capsule and lens movements in rhesus monkeys of various ages. Invest $O p h$ thalmol Vis Sci. 2008;49(12):5484-5494.

11. Croft MA, Glasser A, Heatley G, et al. Accommodative ciliary body and lens function in rhesus monkeys I: normal lens, zonule and ciliary process configuration in the iridectomized eye. Invest $O p b$ thalmol Vis Sci. 2006;47(3):1076-1086.

12. Croft MA, McDonald JP, Nadkarni NV, et al. Age-related changes in centripetal ciliary body movement relative to centripetal lens movement in monkeys. Exp Eye Res. 2009;89(6):824-832.

13. Tamm E, Croft MA, Jungkunz W, et al. Age-related loss of ciliary muscle mobility in the rhesus monkey. Arch Ophthalmol. 1992; 110(6):871-876.

14. Lütjen-Drecoll E, Kaufman PL, Wasielewski R, Ting-Li L, Croft MA. Morphology and accommodative function of the vitreous zonule in 
human and monkey eyes. Invest Ophthalmol Vis Sci. 2010;51(3) 1554-1564

15. Strenk SA, Strenk LM, Guo S. Magnetic resonance imaging of the anteroposterior position and thickness of the aging, accommodating, phakic and pseudophakic ciliary muscle. J Cataract Refract Surg. 2010;36:235-241.

16. Baikoff G, Lutun E, Wei J, Ferraz C. Anterior chamber optical coherence tomography study of human natural accommodation in a 19-year-old albino. J Cataract Refract Surg. 2004;30(3):696-701.

17. Sheppard AL, Davies LN. In vivo analysis of ciliary muscle morphologic changes with accommodation and axial ametropia. Invest Ophthalmol Vis Sci. 2010;51:6882-6889.

18. Wolffsohn JS, Davies LN. Advances in anterior segment imaging. Curr Opin Ophthalmol. 2007;18(1):32-38.

19. Moffat BA, Atchison DA, Pope JM. Age-related changes in refractive index distribution and power of the human lens as measured by magnetic resonance micro imaging in vitro. Vision Res. 2002;42: 1683-1693.

20. Moffat BA, Atchison DA, Pope JM. Explanation of the lens paradox. Optom Vis Sci. 2002;79:148-150.

21. Jones CE, Pope JM. Measuring optical properties of an eye lens using magnetic resonance imaging. Magn Reson Imaging. 2004; 22:211-220

22. Jones CE, Atchison DA, Meder R, Pope JM. Refractive index distribution and optical properties of the isolated human lens measured using magnetic resonance imaging. Vision Res. 2005;45:23522366.

23. Kasthurirangan S, Markwell EL, Atchison DA, Pope JM. In vivo study of changes in refractive index distribution in the human crystalline lens with age and accommodation. Invest Ophthalmol Vis Sci. 2008;49:2531-2540.

24. Helmholtz H. Uber die Akkommodation des Auges. Arch Ophthalmol. 1855;1:1-74.

25. Strenk SA, Strenk LM, Semmlow JL, De Marco JK. Magnetic resonance imaging study of the effects of age and accommodation on the human lens cross-sectional area. Invest Ophthalmol Vis Sci. 2004;45:539-545.

26. Judge SJ, Burd HJ. The MRI data of Strenk et al. do not suggest lens compression in the unaccommodated state (E-letter). Invest $\mathrm{Oph}$ thalmol Vis Sci. 2004;45:539.

27. Hermans EA, Pouwels PJW, Dubbelman M, Kuijer JPA, van der Heijde RGL, Heethar RM. Constant volume of the human lens and decrease in surface area of the capsular bag during accommodation: an MRI and Scheimpflug study. Invest Ophthalmol Vis Sci. 2009;50:281-289.

28. Schachar RA, Kamangar F, Pierscionek BK. To the editor: changes in lens dimensions and refractive index with age and accommodation. Optom Vis Sci. 2008;85:281-282.

29. Drexler W, Findl O, Schmetterer L, Hitzenberger CK, Fercher AF. Eye elongation during accommodation in humans: differences between emmetropes and myopes. Invest Ophthalmol Vis Sci. 1998; 39:2140-2147.

30. Ziebarth NM, Manns F, Uhlhorn S, Venkatraman AS, Parel JM. Non-contact optical measurement of lens capsule thickness in human, monkey and rabbit post-mortem eyes. Invest Ophthalmol Vis Sci. 2005;46:1690-1697.

31. Mallen EAH, Kashyap P, Hampson KM. Transient axial length change during the accommodation response in young adults. Invest Ophthalmol Vis Sci. 2006;47:1251-1254

32. Gerometta R, Zamudio AC, Escobar DP, Candia OA. Volume change of the ocular lens during accommodation. Am J PhysiolCell Physiol. 2007;293:C797-C804.

33. Zamudio AC, Candia OA, Kong CW, Wu B, Gerometta R. Surface change of the mammalian lens during accommodation. Am J Physiol-Cell Physiol. 2008;294:C1430-C1435.

34. Singh KD, Logan NS, Gilmartin B. Three-dimensional modeling of the human eye based on magnetic resonance imaging. Invest Ophthalmol Vis Sci. 2006;47:2272-2279.

35. Goodall N, Kisiswa L, Prashar A, et al. 3-Dimensional modelling of chick embryo eye development and growth using high resolution magnetic resonance imaging. Exp Eye Res. 2009;89:511-521.
36. Liney GP. Magnetic resonance imaging physics lectures. Available at: http://www.hull.ac.uk/mri/lectures/gpl_page.html. Accessed October 2010.

37. Liney GP. MRI from A to Z: A Definitive Guide for Medical Professionals. Cambridge: Cambridge University Press; 2005.

38. Lou L. Troxler effect with dichoptic stimulus presentations: evidence for binocular inhibitory summation and interocular suppression. Vision Res. 2008;48:1514-1521.

39. Millodot M. Dictionary of Optometry and Visual Science. Oxford: Butterworth-Heinemann Elsevier; 2009.

40. Sheppard AL, Davies LN. Clinical evaluation of the Grand Seiko Auto Ref/Keratometer WAM-5500. Ophthalmic Physiol Opt. 2010; 30:143-151

41. Stark LR, Atchison DA. Subject instructions and methods of target presentation in accommodation research. Invest Ophthalmol Vis Sci. 1994;35:528-537.

42. Radhakrishnan H, Charman WN. Age-related changes in static accommodation and accommodative miosis. Ophthalmic Physiol Opt. 2007;27:342-352

43. Santodomingo-Rubido J, Mallen EAH, Gilmartin B, Wolffsohn JS. A new non-contact device for ocular biometry. Br J Ophthalmol. 2002;86:458 - 462 .

44. Dubbleman M, van der Heijde GL, Weeber HA. Change in shape of the aging human crystalline lens with accommodation. Vision Res. 2005; $45: 117-132$.

45. Richdale K, Bullimore MA, Zadnik K. Lens thickness with age and accommodation by optical coherence tomography. Ophthalmic Physiol Opt. 2008;28:441-447.

46. Kirschkamp T, Dunne MCM, Barry JC. Phakometric measurement of ocular surface radii of curvature, axial separations and alignment in relaxed and accommodated human eyes. Ophthalmic Physiol Opt. 2004;24:65-73.

47. Levy NS. Comparing MRIs with movement artifact (E-letter). Invest Ophthalmol Vis Sci. 2000. Available at: http://www.iovs.org/ content/40/6/1162/reply\#iovs_el_7?sid=b42e2405-bb51-4da0-800709b683d2c7c9. Accessed June 10, 2010.

48. Schachar RA, Cudmore DP, Torti R, Black TD, Huang T. A physical model demonstrating Schachar hypothesis of accommodation. Ann Ophthalmol. 1994;26:4-9.

49. Schachar RA. The mechanism of accommodation and presbyopia. Int Ophthalmol Clin. 2006;46:39-61.

50. Goss DA, van Veen HG, Rainey BB, Feng B. Ocular components measured by keratometry, phakometry and ultrasonography in emmetropic and myopic optometry students. Optom Vis Sci. 1997; 74:489- 495.

51. Dubbelman M, van der Heijde GL. The shape of the aging human lens: curvature, equivalent refractive index and the lens paradox Vision Res. 2001;41:1867-1877.

52. Brown N. The change in lens curvature with age. Exp Eye Res. 1974;19:175-183.

53. Olbert D. Problems of lens biometry by Scheimpflug photos. Lens Res. 1988;5:73-81.

54. Davies LN, Dunne MCM, Gibson GA, Wolffsohn JS. Vergence analysis reveals the influence of axial distances on accommodation with age and ametropia. Ophthalmic Physiol Opt. 2010;20:371378.

55. Koretz JF, Cook CA, Kaufman PL. Aging of the human lens: changes in lens shape upon accommodation and with accommodative loss. J Opt Soc Am A Opt Image Sci Vis. 2002;19:144-151.

56. Garner LF, Yap MK. Changes in ocular dimensions and refraction with accommodation. Ophthalmic Physiol Opt. 1997;17:12-17.

57. Weale RA. Presbyopia toward the end of the 20th century. Surv Ophthalmol. 1989;34:15-30.

58. Fincham EF. The mechanism of accommodation. Br J Ophthalmol Supplement. 1937;8:5-80.

59. Atchison DA, Claydon CA, Irwin SE. Amplitude of accommodation for different head positions and different directions of eye gaze. Optom Vis Sci. 1994;71:339-345. 